\title{
The Role of Left Ventrolateral Prefrontal Cortex during Episodic Decisions: Semantic Elaboration or Resolution of Episodic Interference?
}

\author{
Sanghoon Han', Akira R. O'Connor ${ }^{2}$, Andrea N. Eslick ${ }^{3}$, \\ and Ian G. Dobbins ${ }^{4}$
}

\begin{abstract}
Context memory retrieval tasks often implicate the left ventrolateral pFC (LVPFC) during functional imaging. Although this region has been linked to controlled semantic processing of materials, it may also play a more general role in selecting among competing episodic representations during demanding retrieval tasks. Thus, the LVPFC response during context memory retrieval may reflect either semantic processing of memoranda or adjudication of interfering episodic memories evoked by memoranda. To distinguish between these hypotheses, we contrasted context and item memory retrieval tasks for meaningful and nonmeaningful memoranda using fMRI. Increased LVPFC activation during con-
\end{abstract}

\section{INTRODUCTION}

Although the left ventrolateral pFC (LVPFC) has been implicated during episodic retrieval, particularly in tasks that require recovery of context, the nature of its memory contribution remains unclear. Previously, LVPFC (BA 45/ BA 47) has been implicated in linguistic processing, particularly in the controlled processing of semantic and phonological representations (Thompson-Schill, 2003; Wagner, Paré-Blagoev, Clark, \& Poldrack, 2001; Petersen, Fox, Posner, Mintum, \& Raichle, 1988). Given this literature, LVPFC involvement during context memory arguably reflects semantic elaboration of retrieval probes. For example, Dobbins, Foley, Schacter, and Wagner (2002) posited that, when attempting to retrieve a context memory for a given probe, subjects focus attention on the semantic features of the probe that would have been central had the stimulus in fact been encountered earlier in the candidate context. Such a strategy would be consistent with two influential and related principles in the behavioral memory literature known as encoding specificity (Tulving, 1983) and transfer appropriate processing (Roediger, 1990). Both emphasize that episodic retrieval success critically depends upon the

\footnotetext{
${ }^{1}$ Yonsei University, Seoul, South Korea, ${ }^{2}$ The University of St. Andrews, Scotland, ${ }^{3}$ Duke University, ${ }^{4}$ Washington University in Saint Louis
}

text compared with item memory only occurred for meaningful memory probes. In contrast, even demanding context retrieval for nonmeaningful materials failed to engage LVPFC. These data demonstrate that the activation previously seen during episodic tasks likely reflects semantic processing of the probes during episodic retrieval attempt, not the selection among competing elicited episodic representations. Posterior middle temporal gyrus and the body/head of the caudate demonstrated the same selective response as LVPFC, although resting state functional connectivity analyses suggested that these two regions likely shared separate functional relationships with the LVPFC. match between the features previously processed during encoding and those attended at retrieval.

However, it has also recently been suggested that there is considerable functional specialization in LVPFC. For example, on the basis of their own work and the reviewed literature, Badre and Wagner (2007) concluded that the anterior portion of LVPFC ( $\sim \mathrm{BA} 47 / \mathrm{BA} 45)$ is involved in controlled semantic retrieval operations whereas the posterior region ( $\sim \mathrm{BA} 45 / \mathrm{BA} 44)$ is involved in a general selection mechanism that operates following retrieval and resolves interference among competing semantic, phonological, and, perhaps, episodic representations. Supporting a link between LVPFC and semantic processing, previous fMRI studies of context memory have demonstrated functional connectivity between LVPFC and posterior left middle temporal gyrus (MTG; Dobbins \& Wagner, 2005), leading to the suggestion that controlled semantic processing during retrieval attempt is manifested in the interaction of the two regions via inferior fiber pathways (Binder, Desai, Graves, \& Conant, 2009) and consistent with fMRI research linking both regions to tasks requiring controlled or deliberative semantic judgments (Thompson-Schill, D’Esposito, Aguirre, \& Farah, 1997; Vandenberghe, Price, Wise, Josephs, \& Frackowiak, 1996). From this perspective, LVPFC may be critical for biasing or foregrounding the task-relevant semantic representations in posterior MTG during the retrieval attempt, and this interpretation 
is also plausible in light of nonhuman primate anatomical tracing, demonstrating anatomical connectivity between areas TEO and TE and Macaque Area 45 (Gerbella, Belmalih, Borra, Rozzi, \& Luppino, 2010; Webster, Bachevalier, \& Ungerleider, 1994). However, although some evidence exists for functional specialization along the LVPFC axis, other accounts have instead focused primarily on a general selection account with no clear subspecialization of LVPFC as a function of the types of representations that are competing during the task (Gold \& Buckner, 2002). Thus, the region may be generally engaged whenever semantic, phonological, and, perhaps, episodic representations compete for selection.

The possibility that LVPFC may be broadly involved in interference resolution bears critically on the functional interpretation of the LVPFC activation seen during context memory research and brings into question the interpretation of this activity purely in terms of semantic processing. Instead, this activity might represent selection among the competing episodic representations evoked during context memory tasks, not semantic processing of the probes. More specifically, under this selection hypothesis, LVPFC would contribute to context memory retrieval by selecting or differentially biasing the correct episodic information in the face of episodic interference from other similar events. This fits well with the repeated finding that context, but not item, memory tasks evoke an LVPFC response (Dobbins \& Han, 2006; Dobbins \& Wagner, 2005; Dobbins et al., 2002). Because context memory retrieval demands are often highly specific, episodic traces from the same study session will often share considerable features, hence increasingly compete, requiring interference resolution. In contrast, because item recognition can be based on global familiarity, without regards to the specifics of prior individual encounters, episodic trace selection demands are arguably minimal.

In the current report, we specifically test whether LVPFC activation during context retrieval reflects semantic processing of probes or selection among competing episodic representations by comparing context memory using meaningful (scenic pictures) versus nonmeaningful stimuli (fractal-like pictures), and comparing this to item memory judgments with these same materials. If activation reflects selection among competing retrieved episodic memories, then one would expect increased LVPFC activation during context versus item memory tasks, regardless of the format of the memory probes (meaningful vs. nonmeaningful). In contrast, if the activation previously observed in the literature reflects the controlled semantic processing of the probes to facilitate context retrieval, then increased activation should be observed for context compared with item memory, but only for meaningful materials. In the case of nonmeaningful materials, semantic processing would be largely impossible during context memory trials, hence activation should be uniformly low during both context trials and item memory trials.
The current design closely adhered to a three-alternative forced-choice format that has previously yielded strongly left-lateralized activation increases for context versus item memory tasks with meaningful pictorial and verbal stimuli (Dobbins \& Han, 2006; Dobbins \& Wagner, 2005; Dobbins et al., 2002). During encoding, subjects render one of two judgments for each stimulus (pleasant/unpleasant or complex/simple judgment), with these tasks forming the contexts later targeted for retrieval. Following encoding, subjects receive probe triplets consisting of a new item and an encoded item from each context. Context memory trials require selection of the triplet member associated with a specific prior processing task. Item memory trials, instead, simply require the subject to select the novel item and thus recovery of prior context information is unnecessary. A schematic of the procedure and sample picture stimuli for each condition are illustrated in Figure 1. The current design again contrasts context and item memory but extends the manipulation across both meaningful and nonmeaningful materials.

\section{METHODS}

\section{Participants}

Fifteen volunteers (10 women, 18-35 years old) were paid $\$ 40$ for participating. Three additional subjects were excluded from analysis: two for behavioral performance below chance level and one for failure to complete the experiment. Subjects were also screened for color vision. Informed consent was obtained in compliance with the institutional review board at Duke University.

\section{Study Materials}

A total of 270 color photographs of common scenes were drawn from the software Art Explosion (HALLoGRAM Publishing, Aurora, CO). Fractal patterns were similar to those in prior research (Law et al., 2005; Miyashita, Higuchi, Sakai, \& Masui, 1991). These were created using kaleidoscopic rendering software applied to the same stimuli serving as meaningful probe stimuli to preserve basic color characteristics across meaningful and nonmeaningful materials (Photoechos, www.photoechoes.com/). As shown in Figure 1, the software produces stimuli in which it is difficult, if not impossible, to discern any meaningful content. Critically, the experimental design does not require complete elimination of all possible semantic content (although this appears to have been achieved) but merely a considerable reduction, such that one can assume that the retrieval of semantic content is considerably less likely with the nonmeaningful versus meaningful probes. Figure 1 suggests that this goal was easily achieved. Five hundred forty images were used in the design, and all images were divided into 12 lists of 45 images each (six lists consisted of meaningful images and six consisted of nonmeaningful images). Of the 12 total lists, only 8 (four meaningful and 
Figure 1. Schematic figure of experimental paradigm and stimuli example. During encoding phase, participants rated serially presented color pictures on either one of two characteristics (pleasantness or complexity). During immediate retrieval phase, triplets were presented horizontally on the screen and participants made memory discrimination about which item of each triplet was associated with a particular prior classification task (Pleasant Task?, Complex Task?) or which of the three was novel (New Item?). Kaleidoscopic rendering was applied to create nonmeaningful images for the purpose of drastically minimizing participants strategy to adopt a semantic elaboration relative to the unaltered materials.

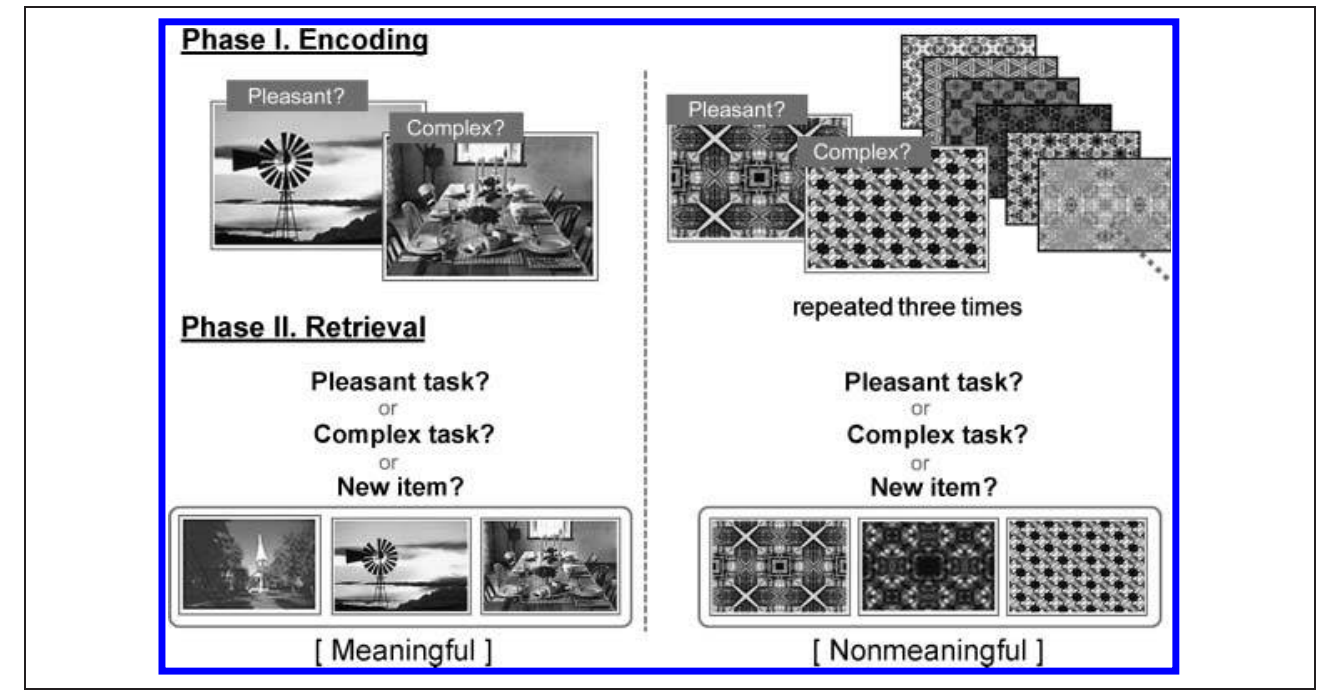

four nonmeaningful) were used for a given subject; the other four lists were reserved in the event of a computer or program malfunction. All lists were randomized for each subject, such that each photo had an equal chance of appearing with each possible study task or as a novel item.

\section{Procedure}

The experiment consisted of eight study-test cycles, four meaningful and four nonmeaningful, the order of which alternated and was counterbalanced across subjects. Each of the study and test cycles was scanned in a separate run. During the study, items were presented with a study cue (Pleasant?; Complex?) and subjects had 4 sec to make their judgment on a 4-point scale: "Very UnpleasantUnpleasant-Pleasant-Very Pleasant" or "Very SimpleSimple-Complex-Very Complex". Subjects studied 30 pictures during each study phase, 15 of which appeared with the "Pleasant?" cue and the other 15 appeared with the "Complex?" cue. The order and assignment of these cues to pictures was randomized for each subject, and study trials were intermixed with six equal length fixation trials per each run. Responding was via fiber-optic keypad using the left hand. Stimuli remained on the screen for $4 \mathrm{sec}$ regardless of RT. The study phase for nonmeaningful items was identical, except that the list was repeated three times given laboratory pilot data and prior behavioral research, demonstrating that increased rehearsal is required for satisfactory encoding of nonmeaningful materials (e.g., Paivio, 1991). During repetitions, items were paired with the same orienting question; however, the presentation order within each list was randomized.
A test phase followed each study phase (one cycle); each test consisted of 15 trials. On each trial, a triplet was presented horizontally below one of three possible test cues (“Pleasant Task?"; "Complex Task?"; or "New Item?”). The test triplets contained two studied and one novel picture - one old item had been encoded via Pleasantness rating task, the other via the Complexity rating task. Subjects were instructed to choose the picture associated with the prior context indicated by the test cue (or to chose the novel item if given the "New Item?" cue). Five of the 15 test trials required recovery of prior pleasantness task performance ("Pleasant Task?"), five recovery of the prior complexity judgment ("Complex Task?"), and five required selecting the novel item of the triplet ("New Item?"). Overall this resulted in 20 trials per each retrieval cue condition (five per each cue across with four scans for meaningful materials and four scans for nonmeaningful materials). Subjects had $6 \mathrm{sec}$ to respond during memory tests with the items remaining on screen for $6 \mathrm{sec}$ regardless of their RT. The order of test trial types was pseudorandomized for each subject, with five intermixed passive fixation trials. The order was determined using an optimal sequencing algorithm (Wager \& Nichols, 2003) designed to facilitate recovery of the BOLD signal. Subjects responded using the ring, middle, and index finger of the left hand, which corresponded to the screen location of the probe picture (left, middle, or right) they wished to select.

\section{fMRI Acquisition}

Scanning was performed on a 3-T General Electric (Waukesha, WI) scanner using a standard head coil. 
Functional data were acquired by using a gradient-echo planar pulse sequence (repetition time $=2000 \mathrm{msec}$, $\mathrm{TE}=31 \mathrm{msec}, 34$ axial slices parallel to the AC-PC plane with near-isotropic voxels of $3.75 \times 3.75 \times 3.8 \mathrm{~mm}$, no gap, interleaved collection). Before functional data collection, four dummy volumes were discarded to allow for equilibration effects. Participants' head motion was minimized by using foam padding. High-resolution T1weighted anatomical images (3-D spoiled gradient recalled acquisition) were also acquired for visualization.

\section{fMRI Analyses Methods}

\section{Event-related $f M R I$}

Analyses focused on the memory retrieval scans. Data were processed using SPM2 (Wellcome Department of Cognitive Neurology, London, U.K.). Slice acquisition timing was corrected by resampling all slices in time relative to the middle slice collected, followed by rigid body motion correction across all runs. Functional data were spatially normalized to a canonical EPI template using a 12-parameter affine and nonlinear cosine transformation, with volumes then resampled into 2-mm isotropic cubes and then spatially smoothed with an 8-mm FWHM isotropic Gaussian kernel. Each scanning session was rescaled, such that the mean global signal was 100 across the volumes. For the analyses, volumes were treated as a temporally correlated time series and modeled by convolving a canonical hemodynamic response function and its temporal derivative with a delta function marking each trial onset. All incorrect responses were modeled as a regressor of no interest, and all contrasts were restricted to correct trials. The resulting functions were used as covariates in a general linear model, along with a basis set of cosine functions that were used to high-pass filter the data and a covariate representing session effects. The least squares parameter estimates of the best-fitting synthetic hemodynamic response function for each condition of interest (averaged across runs) were used in pairwise contrasts and stored as a separate image for each subject. These difference images were then tested against the null hypothesis of no difference between contrast conditions using one-tailed $t$ tests. The data were statistically analyzed, treating subjects as a random effect. Unless stated otherwise, effects were considered significant if they exceeded a voxel-wise threshold of $p<.001$ and consisted of five or more contiguous voxels.

Functional ROIs were extracted by MarsBar Toolbox (Brett, Anton, Valabregue, \& Poline, 2002) from the maps satisfying the above statistical criteria using peristimulus time averaging. Percent signal averages were obtained for the significant voxels within an 8- $\mathrm{mm}$ radius of each of the SPM-identified maxima and further analyzed using off-line statistical software. The implicit baseline response of each voxel reflects the constant term in the least squares linear model describing that voxel's response during the session, and the activity of each voxel was scaled, such that the constant was equivalent to 100 for each session. Thus, departures from this value reflect percentage changes relative to a baseline or constant activation level for the sessions.

\section{Resting State Connectivity MRI}

To further characterize the potential functional role of LVPFC, functional connectivity analysis using resting state functional MRI data in an independent group of 39 righthanded participants was also examined. These data were collected at Washington University in St. Louis with informed consent obtained in a manner approved by the institutional review board. This analysis was motivated by the unexpected finding of a caudate activation that responded to the experimental manipulation in the same qualitative manner as LVPFC and posterior MTG (see Amplitude Analyses section). Given that this region is not traditionally associated with semantic processing, the resting state functional connectivity analysis was conducted to determine if the pattern of spontaneous activity at rest suggested that LVPFC, posterior MTG, and caudate regions were all similarly intercorrelated or whether the pattern of correlation instead suggested separate unique relationships between LVPFC and the two other regions. Although the precise basis of the slow oscillatory intrinsic activity $(<.1 \mathrm{~Hz})$, that is, the basis of resting state connectivity analysis remain unclear, there is consensus that the procedure captures mono- and polysynaptic anatomical connectivity between brain regions (Van Dijk et al., 2010). Furthermore, because the activity is intrinsic and not driven by external transients such as an experimental task, the method avoids mischaracterizing regions as functionally connected merely because they respond to external task manipulations. For example, both motor and visual regions would be expected to respond to an external cue during a go/no-go task, but this joint increase in amplitude is not a marker of anatomical connectivity. Instead it merely reflects that different networks will often be jointly recruited during complex tasks.

Resting connectivity data were collected on a 3-T Siemens Trio whole-body MRI scanner (Siemens Medical Solutions, Erlangen, Germany) using a standard whole-head coil. Data were acquired over two 6-min scans, which used an interleaved ascending echo-planar pulse sequence (164 volumes, repetition time $=2200 \mathrm{msec}, \mathrm{TE}=27 \mathrm{msec}$, 36 axial slices parallel to the AC-PC plane with isotropic 4-mm voxels, no interslice gap). The first two volumes of each scan were discarded to allow for T1 equilibration. Head motion was minimized using foam padding. Highresolution T1-weighted and T2-weighted anatomical images were acquired before resting connectivity scans, and functional scans of unrelated tasks (not discussed) were conducted after resting connectivity scans. Participants fixated on a cross for the duration of each 6-min resting connectivity scan. 
Connectivity data were processed with SPM5 (Wellcome Department of Imaging Neuroscience, London, U.K.). Slice acquisition timing correction was carried out by temporally resampling relative to the middle slice collected, followed by rigid body motion correction. Functional volumes were then spatially normalized to a canonical echo-planar template using 12-parameter affine and cosine basis transformations and resampled to 3-mm isotropic voxels. Volumes were then spatially smoothed with a 6-mm Gaussian kernel.

Single-seed connectivity maps were generated by modeling 18 sources of nonspecific variance (six movement parameters, signal from spheres in the left lateral ventricle, in left hemisphere deep cerebral white matter and averaged across the whole brain, and the nine first derivatives of these parameters) alongside the covariate of interest, which consisted the extracted time series from ROIs determined based on the event-related fMRI amplitude analysis (see above; e.g., Fox et al., 2005). Resulting maps were subjected to the same threshold as in the Amplitude Analyses above $(p<.001,5$ voxels). Statistically, subjects were then treated as a random effect, meaning that simple seed connectivity maps illustrate areas whose activation reliably covaries with the seed region, on a scan-by-scan basis, after nonspecific effects have been controlled.

\section{RESULTS}

\section{Behavioral Data}

Behavioral data for correct responses were analyzed using repeated measures ANOVA. Follow-up pairwise comparisons used Fisher's LSD test at an alpha of .05.

\section{Accuracy}

An ANOVA for accuracy with factors of Task (pleasant, complex, novel) and Materials (meaningful, nonmeaningful) revealed a main effect of Task $(F(2,28)=9.97, p<$ $.001)$ and Materials $(F(1,14)=6.95, p<.05)$, with no interaction between these factors $(F(2,28)=2.28, p>.12)$. The main effect of Materials resulted from the generally lower accuracy for nonmeaningful materials $(M=0.83)$ compared with meaningful materials $(M=0.90)$; however, performance during the former was still quite good. Sim- ple effects analysis demonstrated that the main effect of Task reflected the fact that novelty detection was easier $(M=0.92)$ than pleasantness $(M=0.85)$ and complexity $(M=0.83)$ context retrieval, with the two context tasks not significantly differing in difficulty (see Table 1).

\section{RTS}

RT data were analyzed for correct responses only. An ANOVA of RT with factors of task (pleasant, complex, novel) and materials (meaningful, nonmeaningful) yielded a main effect of Task $(F(2,28)=13.40, p<.001)$ and Materials $(F(1,14)=6.93, p<.05)$. Simple effects analysis demonstrated that the main effect of Task reflected the fact that novelty detection $(M=2555 \mathrm{msec})$ was significantly quicker than the pleasantness $(M=2824 \mathrm{msec})$ and complexity ( $M=2939 \mathrm{msec})$ context retrieval tasks, which did not differ from one another. The main effect of the Stimulus Type was conditioned by a Stimulus Type $\times$ Cue Type interaction $(F(2,28)=10.79, p<.001)$. RTs were not significantly affected by the stimulus type during the pleasant and complex context retrieval tasks. However, subjects were significantly quicker during the novelty judgment task for meaningful ( $M=2400 \mathrm{msec})$ compared with nonmeaningful stimuli $(M=2710 \mathrm{msec})$.

\section{fMRI}

\section{Amplitude Analyses}

Context versus item memory retrieval contrasts are shown in Figure 2 for meaningful and nonmeaningful materials. There are clear differences in activation patterns that are likely driven by the differences in the materials (Figure 2, top vs. bottom). For meaningful materials, this contrast implicated left rostrolateral, ventrolateral and premotor pFC, in addition to the medial pre-SMA, bilateral caudate, precuneus, left inferior parietal lobule and left posterior MTG (Figure 2, top). In contrast, the extent of activation was greatly reduced with nonmeaningful materials, and the entire LVPFC surface was eliminated from the map (Figure 2, bottom).

To examine the LVPFC response more carefully, time courses were extracted from unbiased anterior, middle, and posterior ROIs along the left ventrolateral surface

Table 1. Mean Proportion Correct and RTs (in msec) as a Function of Cue Type (Question Type)

\begin{tabular}{|c|c|c|c|c|c|c|c|c|}
\hline \multirow[b]{3}{*}{ Cuе Tyре } & \multicolumn{4}{|c|}{ Proportion Correct } & \multicolumn{4}{|c|}{$R T s$} \\
\hline & \multicolumn{2}{|c|}{ Meaningful } & \multicolumn{2}{|c|}{ Nonmeaningful } & \multicolumn{2}{|c|}{ Meaningful } & \multicolumn{2}{|c|}{ Nonmeaningful } \\
\hline & $M$ & $S D$ & $M$ & $S D$ & $M$ & $S D$ & $M$ & $S D$ \\
\hline Pleasant? & 0.89 & 0.09 & 0.82 & 0.15 & 2866.33 & 495.70 & 2781.75 & 482.94 \\
\hline Complex? & 0.88 & 0.09 & 0.78 & 0.16 & 2891.98 & 520.09 & 2987.78 & 461.95 \\
\hline Novel? & 0.93 & 0.08 & 0.91 & 0.10 & 2399.94 & 367.87 & 2710.59 & 474.27 \\
\hline
\end{tabular}


Figure 2. Contrasts of context versus item memory retrieval during meaningful and nonmeaningful probe conditions. Activation maps are overlaid on a canonical brain surface using MRIcron software (www.cabiatl.com/ mricro/). Effect was greatly reduced with nonmeaningful materials, especially in the LVPFC, MTG, and caudate regions (bottom). CTXT $=$ context memory condition ITM $=$ item memory condition

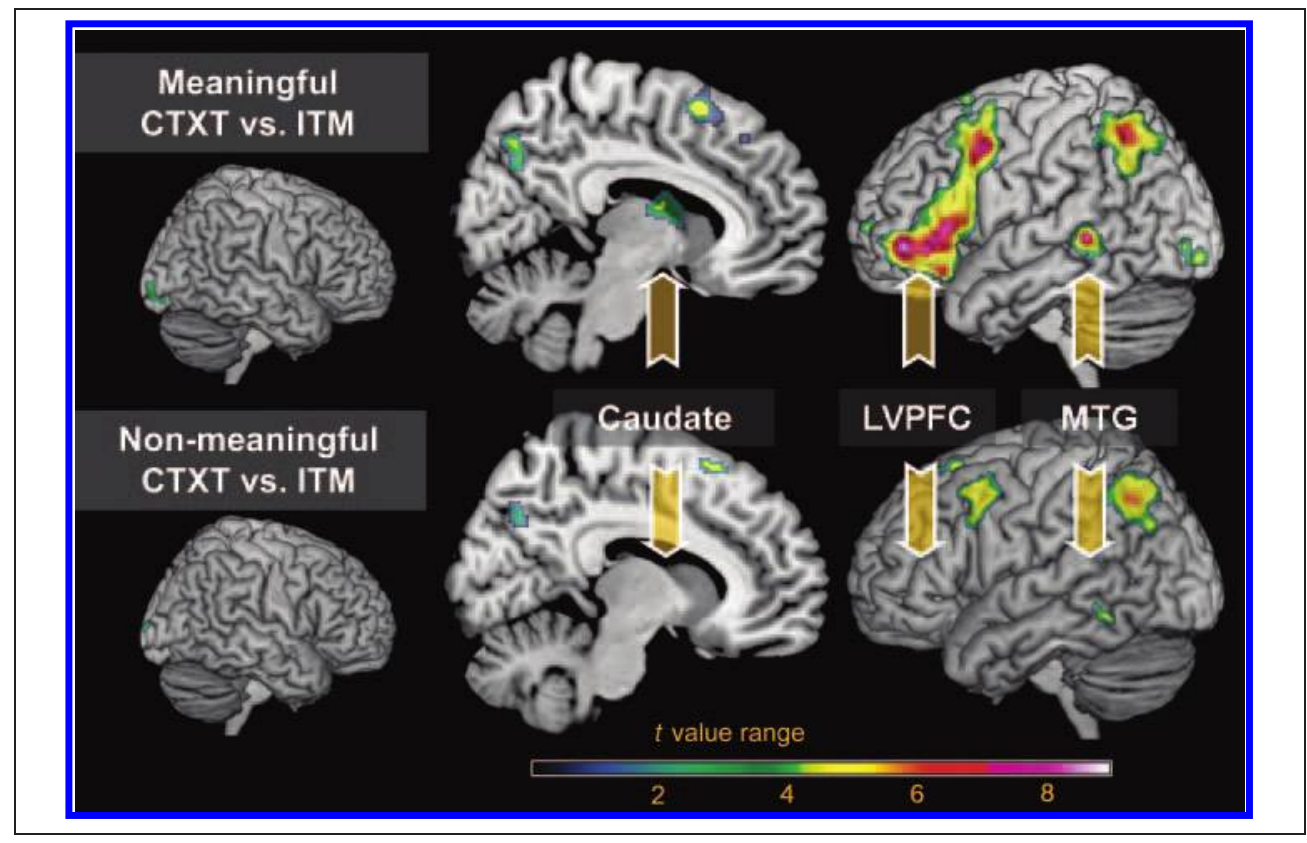

approximately corresponding to BA 10, BA 47, and BA 45, respectively (Figure 3). These ROIs were based on the unbiased contrast of context versus item retrieval trials collapsed across both meaningful and nonmeaningful materials, which implicated the entire LVPFC region. The extracted time courses demonstrate greater recruitment of ventorlateral pFC for both types of context memory tasks (pleasantness and complexity) in comparison with the item memory task, but this differential response only occurs for meaningful materials. This was confirmed using
Stimulus Type (meaningful vs. nonmeaningful) $\times$ Retrieval Condition (pleasant context task, complex context task, item task) ANOVAs with a dependent measure of the summed response across all 10 time points illustrated in Figure 3. This corresponds to a comparison of the area under the BOLD response for all three retrieval conditions across the two material types (i.e., Borst, Taatgen, Stocco, \& van Rijn, 2010). All post hoc pairwise comparisons used the Tukey's HSD correction for family-wise error maintaining a critical alpha of .05.

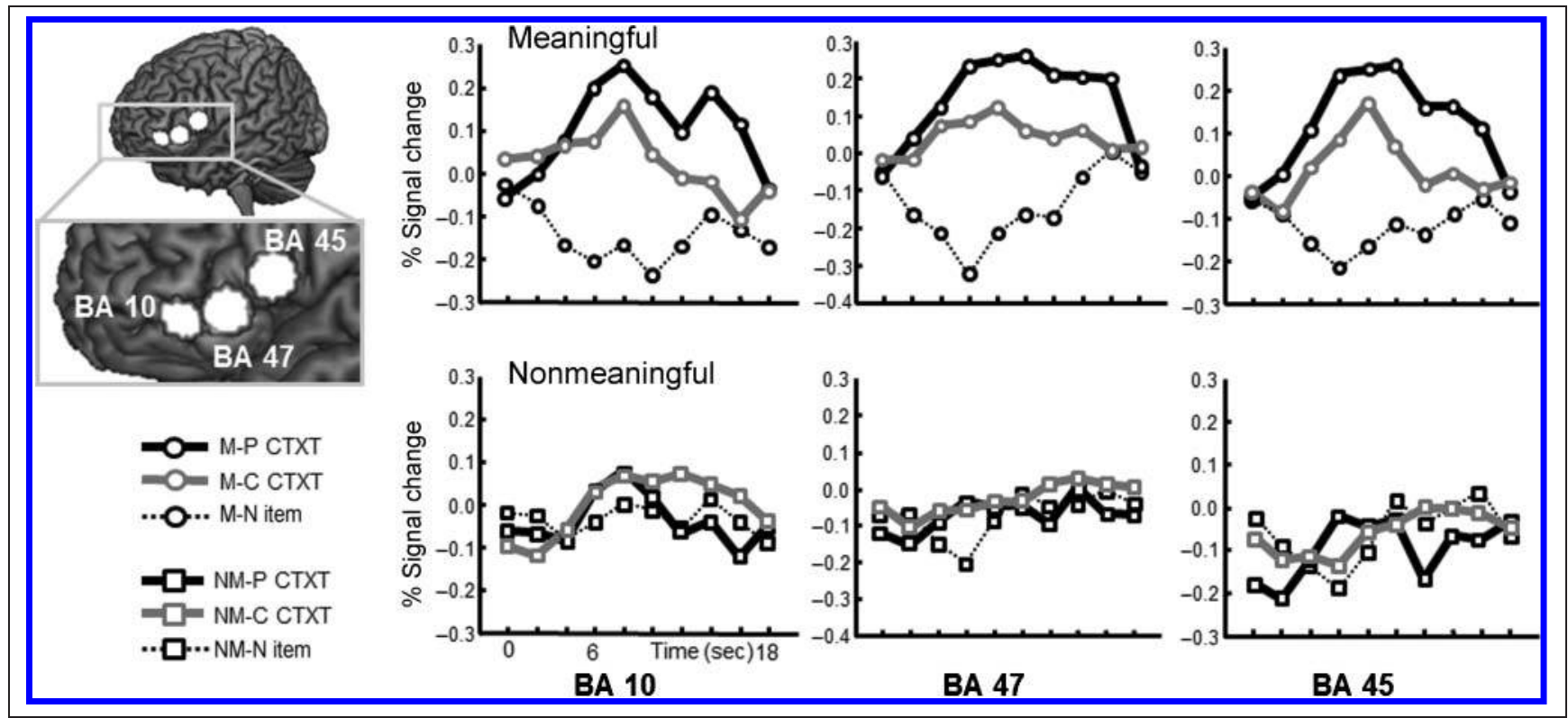

Figure 3. Reconstructed event-related hemodynamic responses from functional ROIs. Three ROIs (BA 10, BA 47, and BA 45) in LVPFC regions were drawn from the map generated in the contrast of context versus item retrieval conditions regardless of the format of the probes (meaningful and nonmeaningful trials). $\mathrm{M}=$ meaningful; $\mathrm{NM}=$ nonmeaningful; $\mathrm{P}=$ pleasant task; $\mathrm{C}=$ complex task; $\mathrm{N}=$ new item; CTXT $=$ context memory; $\mathrm{ITM}=$ item memory. 
In BA 10 (Figure 3, left; $\left[\begin{array}{lll}x y z & z\end{array}\right]=\left[\begin{array}{lll}-40 & 47 & -6\end{array}\right]$ ), there was a significant Stimulus Type $\times$ Retrieval Condition interaction $(F(2,26)=6.15 ; p<.01)$. Follow-up simple effects analyses demonstrated significant signal differences across the retrieval tasks for meaningful stimuli $(F(2,26)=10.46, p<.001)$, but not for nonmeaningful stimuli $(F(2,26)=0.19, p=.83)$. Post hoc comparisons revealed that, for meaningful stimuli, both context tasks evoked greater signal than the item task, although they were not significantly different from one another. BA 47 (Figure 3, center; $\left[\begin{array}{lll}x & y & z\end{array}\right]=\left[\begin{array}{lll}-48 & 35 & -4\end{array}\right]$ ) also yielded a Stimulus Type $\times$ Retrieval Condition interaction $(F(2$, $26)=15.48, p<.001)$. Simple effects analyses again demonstrated significant signal differences across the retrieval tasks for meaningful stimuli $(F(2,26)=14.12$, $p<.001$ ) with no effect present for nonmeaningful stimuli $(F(2,26)=0.33, p=.73)$. For meaningful stimuli, post hoc comparisons demonstrated that both context tasks yielded greater signal than the item task, although they were not significantly different from one another. Finally, BA 45 (Figure 3, right; $\left[\begin{array}{lll}x y z & z\end{array}\right]=\left[\begin{array}{lll}-54 & 21 & 8\end{array}\right]$ ) also yielded a Stimulus Type $\times$ Retrieval Condition interaction $(F(2,26)=6.67, p<.01)$. Simple effects analyses demonstrated significant signal differences across the retrieval tasks for meaningful stimuli $(F(2,26)=8.65, p<.01)$ and with no effect present for nonmeaningful stimuli $(F(2,26)=0.29, p=.75)$. Post hoc comparisons for meaningful materials demonstrated greater activation for the pleasant context task relative to the item task, whereas the complex context task was marginally more active than the item task $(p=.07)$. As in the preceding comparisons, the context tasks did not significantly differ from one another.

Overall the analyses support the conclusion that the LVPFC is differentially activated for context versus item retrieval, but only for materials in which semantic processing is possible, that is, the meaningful stimuli. This supports the hypothesis that the regions are involved in the processing of the retrieval probes' characteristics linked to long-term semantic or linguistic knowledge. In contrast, there was minimal differential activity across the context and item retrieval tasks when the materials were rendered nonmeaningful through kaleidoscopic scrambling. Critically, if episodic interference alone was sufficient for ventrolateral $\mathrm{pFC}$ recruitment, then prominent recruitment should have been observed during the context versus item memory contrast with the nonmeaningful materials. Indeed, the context versus item memory contrast for nonmeaningful materials would be expected to yield a more prominent differential recruitment than for meaningful materials, because the behavioral data demonstrated that the context task was relatively more demanding when using the nonmeaningful stimuli (Table 1), hence was likely associated with greater episodic interference. Instead, the selectivity of the differential response to meaningful materials suggests that subjects respond to challenging episodic demands by strategically processing the probes' semantic characteristics when possible and when transfer is appropriate. We consider the potential adaptive benefit of this response further in the discussion. Before turning to regions outside LVPFC, it is important to note that the above analyses should not be taken to suggest that engagement of LVPFC is equivalent for the two source tasks performed on the meaningful materials. Although the direct comparison of the context tasks was not significant when summed responses of ROIs from the three regions were considered separately, it is clear from Figure 3 that there is a general trend toward greater activation for the pleasant versus complex context tasks during the trials with meaningful materials. This may reflect the fact that judgments of stimulus complexity are likely less dependent upon the semantic characteristics present in the stimulus and can be made in part based on the perceptual complexity present. However, direct comparison of the pleasant context and complex context tasks for the meaningful materials, using a whole-brain analysis, only revealed a difference in BA 45 (MNI coordinate [- $\left.\begin{array}{lll}-50 & 22 & 6\end{array}\right]$ ) with no other regional differences observed. Additionally, the reverse contrast identified only small, isolated clusters in right parietal cortex [42 -58 50], left superior frontal gryrus [ $-22-672]$, and cerebellum showing a greater response for the complex context versus pleasant context source tasks. Given these findings, we refrain from making any strong process distinctions across the two source tasks and instead conclude that both likely involved considerable semantic elaboration of the materials.

Aside from the LVPFC region, two other potential key regions demonstrated greatly reduced or completely eliminated context versus item activation differences for nonmeaningful compared with meaningful materials, namely, left MTG and bilateral body of the caudate (Figures 2 and 4). For the former, an unbiased ROI defined as above (Figure 4 , left) revealed a Stimulus Type $\times$ Retrieval Condition $(F(2,26)=4.75$, MSe $=1.33, p<.05)$ interaction. Simple effects analyses demonstrated a significant effect of task for meaningful materials $(F(2,26)=10.32$, MSe $=$ $1.23, p<.001)$, and no effect of task was present for nonmeaningful materials $(F(2,26)=0.11, p=.90)$. For the meaningful stimuli, the two context conditions did not differ from one another, but both were greater than the item condition.

An unbiased ROI for the caudate region was not available because the context versus item contrast, collapsed across material type, did not implicate this region even at relaxed thresholds. Nonetheless the time courses from the left caudate activation ROI defined using the meaningful trials are shown in Figure 4 (right) because the response was highly selective, and connectivity analyses below establish the functional importance of the region using an independent data set. The region demonstrated a Stimulus Type $\times$ Retrieval Condition interaction $(F(2$, $26)=8.88$, MSe $=0.072, p<.05)$. Simple effects analyses demonstrated a significant effect of task for the meaningful materials $(F(2,26)=9.41$, MSe $=1.29, p<.001)$, 
Figure 4. Extracted hemodynamic responses from MTG and caudate regions. Left caudate ROI was drawn from the meaningful context versus item retrieval contrast. $\mathrm{M}=$ meaningful; $\mathrm{NM}=$ nonmeaningful; $\mathrm{P}=$ pleasant task; $\mathrm{C}=$ complex task; $\mathrm{N}=$ new item; CTXT $=$ context memory; ITM = item memory.

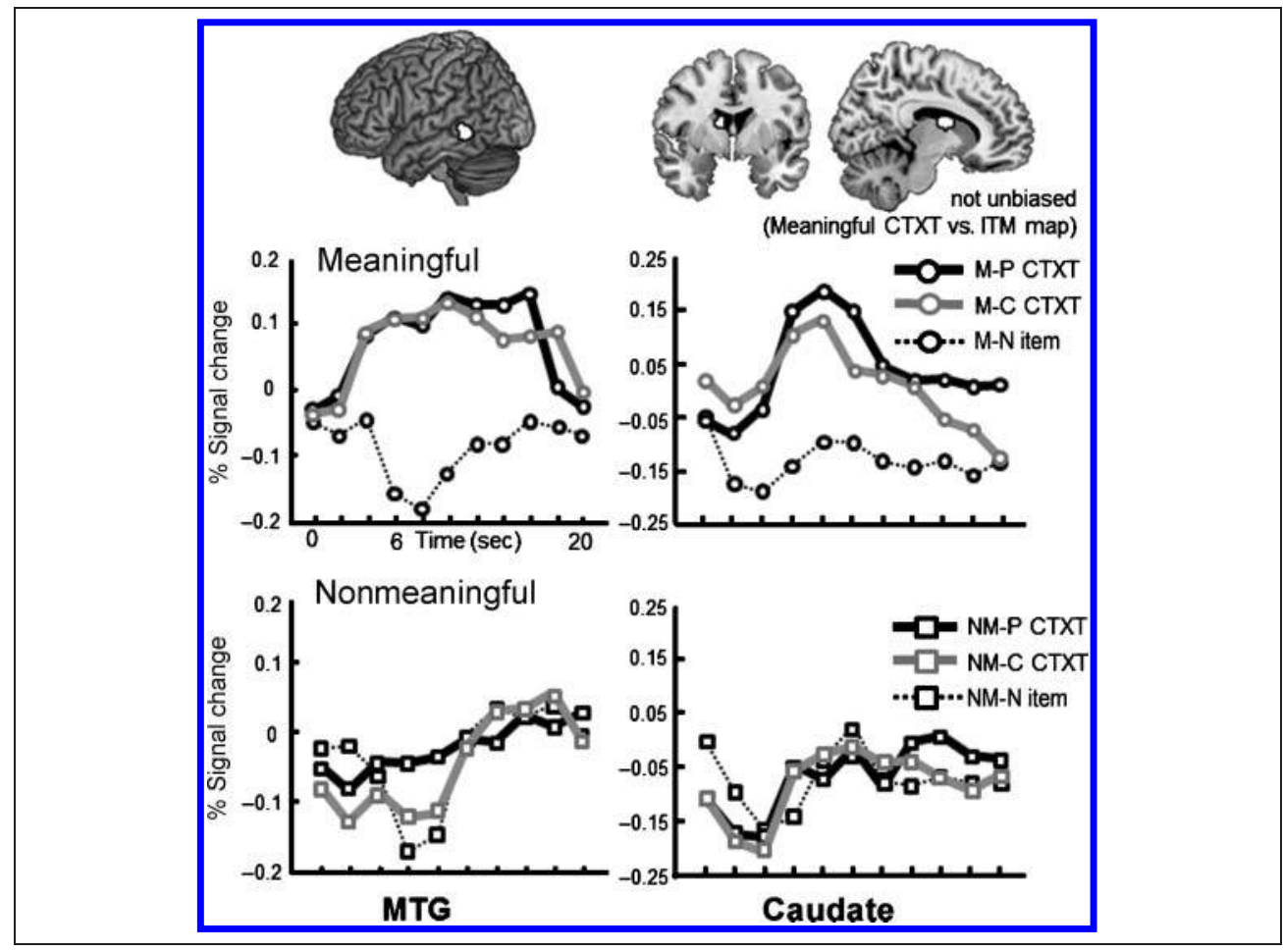

but no effect of task for the nonmeaningful materials $(F(2,26)=0.06, p=.94)$. For the meaningful materials, the context tasks did not differ, but both yielded greater activity than the item task.

Overall, the pattern in the amplitude analysis is clear. There is a prominent recruitment of several cortical areas for context memory compared with item memory. If this recruitment simply reflected the relative difficulty of the two tasks or was a direct response to the presence of episodic interference, then it should have been observed regardless of whether the materials were meaningful or nonmeaningful. In contrast, the differential context memory response was only observed when the materials were meaningful. Thus, although the activation may reflect a strategic response to episodic interference, it is a response that requires the probes have meaningful semantic content. This supports the hypothesis that the observers responded to episodic interference by processing the semantic features of the memory probes when it was possible to do so.

\section{Resting Connectivity Analyses}

One interesting finding of the current study is that the retrieval contrasts also implicated the head/body of caudate, which is not a region typically linked to semantic processing. Interaction between the striatum and $\mathrm{pFC}$, via corticostriato-thalamic loops, is well established (Alexander, DeLong, \& Strick, 1986) and recent resting-state functional connectivity research suggests that the head of the left caudate may interact with inferio-lateral $\mathrm{pFC}$ regions as well as middle frontal gyri (Barnes et al., 2010). Thus, a link between ventrolateral pFC and caudate is plausible and consistent with the caudate forming a functional circuit with pFC that may be critical for some forms of cognitive control. Critically, however, there does not appear to be a link between head/body of the caudate and posterior MTG, although areas TE and TEO in the nonhuman primate do project to the pulvinar nuclei of the thalamus (Webster, Bachevalier, \& Ungerleider, 1993). Overall, this suggests that although these three regions (LVPFC, left head/body of caudate, and left MTG) may show similar amplitude responses to the current manipulations, they may not be part of the same functional network. We examined this possibility with an independent resting state connectivity data set using the three ROIs above. Our initial hypothesis was that the common pattern of activation demonstrated in the caudate and MTG in the event-related data was merely the result of the fact that the active task demands recruited two separable processes, namely, a caudate response possibly associated with working memory updating (Marklund et al., 2009) and an MTG response associated with controlled semantic retrieval or topdown semantic biasing (e.g., Dobbins \& Wagner, 2005). If so, then a resting state connectivity analysis should indicate that these regions are not functionally connected; hence, the similar response profiles in the event-related data were the result of the task recruiting separable cognitive functions.

Each seed region (LVPFC, left caudate, left MTG) was separately entered into a design matrix as a predictor, using the same statistical threshold as the Amplitude Analyses above ( $p<.001,5$ contiguous voxels). As shown in Figure 5, these single seed analyses demonstrate that, at 
rest, the LVPFC implicates both the left caudate and the left MTG (A). In contrast, the left MTG seed implicates the LVPFC, but it does not implicate the caudate (B). Additionally, the failure of MTG to implicate the caudate region was again observed when a very lax threshold $(p<$ $.01,5$ contiguous voxels) was used demonstrating that this is not a simple thresholding phenomenon. Finally, the caudate seed region implicated the LVPFC but did not implicate the left MTG (C), and again, this null finding was confirmed with an increasingly lax threshold $(p<.01$, 5 contiguous voxels). Thus, the simple connectivity maps converge with anatomical connectivity literature discussed above, leading to the conclusion, that although LVPFC may share relationships with the caudate and with the MTG (A), the latter two regions are not functionally connected with one another. In other words, the fact that the caudate and MTG both show a similar response to the experiment conditions in the event-related data is likely because of the fact that each shares a separate functional relationship with LVPFC. Additionally, one unexpected final pattern evident in the resting-state connectivity seed analysis data further underscores the functional distinction between the caudate and MTG ROIs. As shown in Figure 5, the caudate unsurprisingly shares a strong positive correlation with the adjacent globus pallidus and thalamus, the primary output pathway leading back to cortex (C). In contrast, the MTG seed not only fails to share a positive relationship with these areas (B) but, in fact has a significant negative association with the structures $(\mathrm{B}$, inset, blue regions). The data suggest that, as activation spontaneously increases in the MTG, it decreases in the thalamus and globus pallidus, whereas when activation spontaneously increases in the caudate, it also increases in these structures. This is noteworthy given that the nonhuman primate literatures indicate a link between TE and TEO and posterior thalamus/pulvinar in macaque (Webster et al., 1993). This suggests that the caudate and MTG may have largely antagonistic roles with respect to the major output structures of the BG. Overall, then, the resting state functional connectivity analysis suggests that the LVPFC response is linked to two functionally separate regions.

\section{GENERAL DISCUSSION}

The current data yielded two important findings for understanding the role of LVPFC during context memory discrimination. First, the context memory linked LVPFC activation does not reflect selection processes operating directly on interfering episodic memory representations. If this were the case, then the nonmeaningful materials should have also demonstrated a clear context greater than item response given that context retrieval was particularly demanding with nonmeaningful materials. Instead, the differential context greater than item memory activation was only observed using meaningful materials. This indicates that the activation is likely an indirect response to challenging episodic retrieval or episodic interference and is consistent with the hypothesis that subjects respond to episodic interference by more selectively processing the semantic characteristics of the probes when

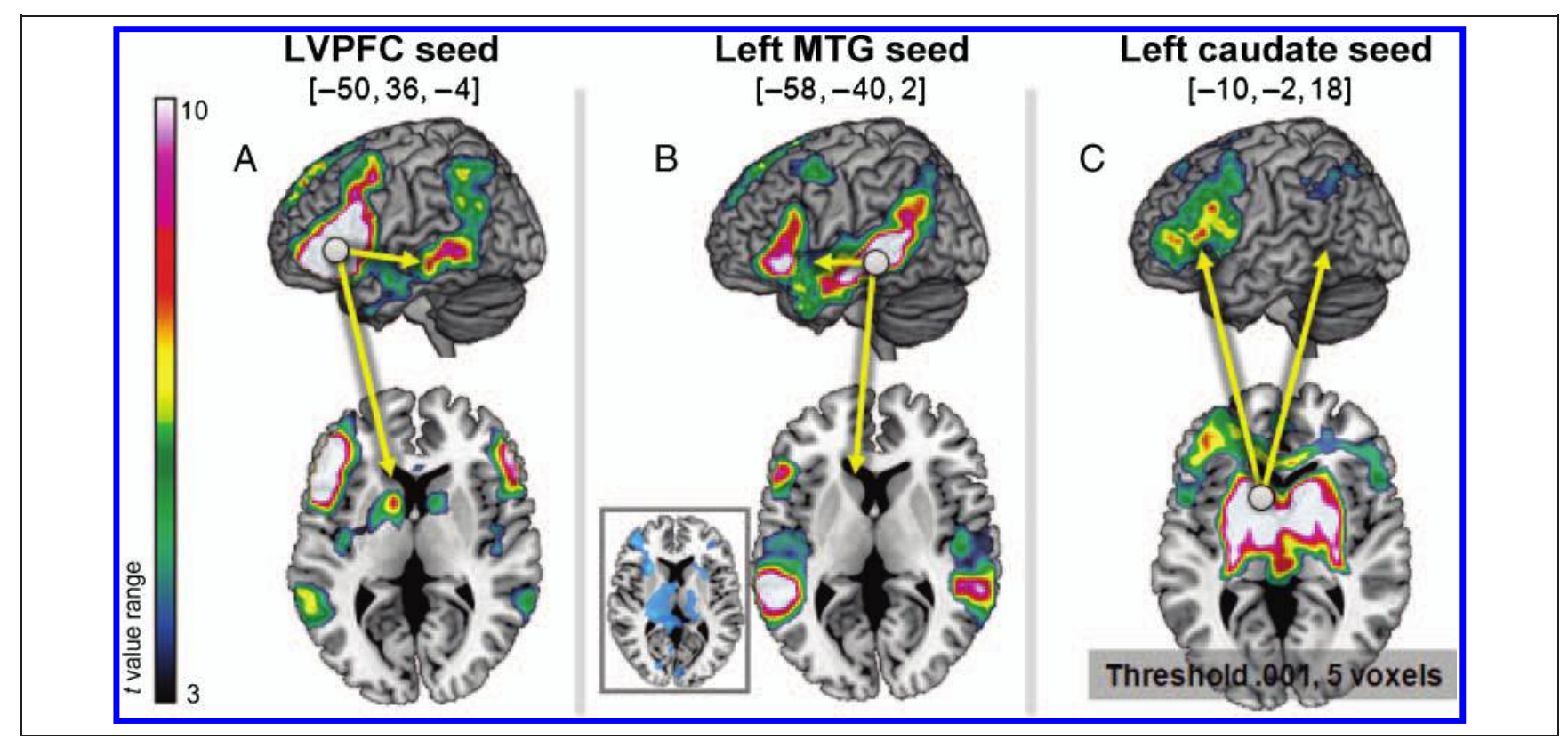

Figure 5. Resting-state seeds and single-seed resting connectivity analyses maps. Resting-state network of regions demonstrating significant activation associated with (A) the LVPFC seed (an 8-mm-diameter sphere; maxima on $[-50,36,-4]$ MNI coordinate), (B) the left MTG seed (an 8-mm-diameter sphere; maxima on $[-58,-40,2]$ ), and (C) the left caudate seed (an 8-mm-diameter sphere; maxima on $[-10,-2,18]$ ). Each ROI used to seed resting-state analyses were circled on the images. B inset (blue regions) indicates a significant negative activation associated with the left MTG activation. 
it is possible to do so. In the cognitive literature, this is often referred to as "semantic elaboration," and it is presumably necessary for foregrounding the subset of semantic features that likely would have been the central focus of processing during the original encoding experience. To the degree participants can successfully restrict attention to the semantic features that were central during the prior candidate task context, the likelihood of diagnostic episodic retrieval is increased (Roediger, 1990; Tulving \& Thomson, 1973) and the potential for interfering episodic retrieval is reduced (Jacoby, Shimizu, Daniels, \& Rhodes, 2005).

Although the semantic elaboration hypothesis is supported, it is important to note that other linguistic representations are also available in the meaningful materials, such as phonological or lexical representations. That said, it is not clear how such features would clearly differentiate the prior encoding operations that are the target of the current context memory tasks, namely, prior judgments of complexity and pleasantness. In other words, it is not clear how focusing on, say, the particular phonological properties of a stimulus would help the observer determine whether it was previously rated using a pleasantness or complexity semantic judgment, because both judgments are presumably linked to the same phonological processing, but different semantic processing.

The second important finding was that the LVPFC is functionally connected with two regions that appear to be functionally independent from one another, namely, the caudate head/body and left posterior MTG. Although all three regions display a similar pattern of mean amplitude in response to the current experimental manipulations, they appear to do so for different functional reasons as revealed by the resting state connectivity analysis. This finding, in part, corroborates previous work linking the ventrolateral $\mathrm{pFC}$ and MTG during controlled semantic retrieval (Cadoret \& Petrides, 2007; Dobbins \& Wagner, 2005). However, we are not aware of prior imaging work, suggesting a key role for the caudate in understanding ventrolateral pFC function during context memory retrieval.

On the basis, in part, of the known role of the striatum in procedural learning and cognitive control (i.e., Grahn, Parkinson, \& Owen, 2008), one working hypothesis is that the caudate may be critical for the selective implementation of semantic elaborative strategies at the trial level, particularly when those strategies are not directly cued from the environment. Such strategies are unnecessary and inefficient for the item retrieval trials because no specific subsets of semantic features are critical for identifying the novel item of the triplet. Additionally, a semantic elaboration strategy is impossible for nonmeaningful stimuli. Instead it is only during context memory queries to meaningful materials that controlled semantic elaboration of the probes should be engaged and only because this strategy presumably improves retrieval outcomes in the long run. This characterization is consistent with frameworks that emphasize a core role for dorsal striatum in the learning and the expression of adaptive instrumental behavior (O'Doherty et al., 2004), with the behavior in this case being the engagement of a semantic elaboration strategy mediated by LVPFC.

Additionally, the framing of semantic elaboration at test as an instrumental behavior engaged to mitigate episodic interference and/or improve contextual retrieval outcomes is consistent with recent findings by Raposo, Han, and Dobbins (2009), examining list discrimination memory. These authors examined the ability to selectively endorse items from an initial study list when potentially interfering words in a second nontarget list were encoded using the same semantic classification task as the initial list (nondistinctive lure processing) versus a new semantic task in the second list (distinctive lure processing). The findings confirmed the prediction that LVPFC would be recruited more heavily in the distinctive condition where it was predicted that subjects would focus attention on the distinctive semantic attributes of the probes related to the initial task. Furthermore, as in the current study, caudate activation was also observed in concert with the LVPFC activation, although the activation was right and not bilateral as in the current data.

A somewhat different interpretation of the caudate activation in the current task is that it critically supports the working memory updating processes tied to the requirement to evaluate the multiple semantic representations of the probes in light of their evoked episodic content. For example, when attempting to select the item associated with the pleasant context source task, one might recover episodic information indicating the item under the current focus of attention was encountered under the other, inappropriate context, namely, the Complexity Context source task. From a working memory updating perspective, this means that this item should be discarded and not considered during the remainder of the trial. Thus, as the trial proceeds, the episodic information (or lack thereof) that is recovered as the probes are sequentially considered will spur various working memory updating requirements (see also Marklund et al., 2009). In short, each probe's targeted semantic representation may require updating throughout the retrieval epoch in terms of the goal relevance of its evoked episodic content.

Critically, under either a strategic elaboration account or a working memory updating account, the caudate response cannot merely reflect motor preparation demands because in all tasks the subjects select one of the three probes via keyboard. Thus, the data clearly demonstrate that the caudate, like the LVPFC, demonstrates a materialdependent response, likely linked to a higher-order cognitive demand. Determining whether this demand may be best construed as reflecting the goal-dependent engagement of a semantic elaboration strategy or requiring working memory updating of multiple semantic representations in light of their elicited remembrances during a trial will require further research. 
Although activation of LVPFC and caudate were highly selective to the combination of context memory retrieval demands and meaningful probes, left dorsal premotor $\mathrm{pFC}$, superior medial $\mathrm{pFC}$ and left parietal regions did not display this specificity. Instead, these areas displayed a significant context versus item memory activation even for nonmeaningful stimuli (see Figure 2), although this differential activation was reduced compared with that seen with meaningful materials. These regions have been implicated in verbal working memory maintenance (Veltman, Rombouts, \& Dolan, 2003), which is consistent with the pattern observed here, because although the nonmeaningful condition materials are likely not verbalizable, the context tasks specified by the retrieval queries are verbalizable. Cognitive and computational models assume that observers must translate these types of retrieval queries into descriptions of the targeted retrieval content that must be held in working memory to respond appropriately should matching episodic content be recovered (e.g., Norman \& Bobrow, 1979). Consistent with this interpretation, Dobbins and Han (2006) observed differential activity in very similar left dorsal premotor (BA 44/BA 6) region that reliably increased with the contextual specificity of the retrieval queries that appeared before the actual retrieval probes. More specifically, this region demonstrated greater activation for context memory queries than for item memory queries, even when no memory probes were present in the environment, leading to the conclusion that it was involved in the maintenance of retrieval descriptions in verbal working memory. Critically, if this region supports general verbal working memory demands, then activation should further increase with the onset of retrieval probes, provided they are also meaningful, because the verbal descriptions of the probes must also be held in working memory during the trial. This, in fact, occurred in Dobbins and Han (2006), in which all probes were meaningful. In the current data, this interpretation fits with the observation that the differential activation is greater for meaningful versus nonmeaningful materials, because the former will have semantic representations for the probes that in addition to the retrieval description, must also be held in working memory until retrieval occurs or the search is terminated.

Although the current findings are largely concordant with prior fMRI research on semantic and episodic retrieval, they did not suggest a functional distinction between the mid and anterior ventrolateral regions (Badre \& Wagner, 2007; Badre, Poldrack, Pare-Blagoev, Insler, \& Wagner, 2005; Dobbins \& Wagner, 2005). As noted in the Introduction, recent research has suggested the anterior region $(\sim \mathrm{BA} 47)$ may be more selectively involved in controlled semantic retrieval whereas the more posterior region $(\sim \mathrm{BA} 45)$ may serve a more general selection role, resolving interference between representations from several different domains such as phonological, lexical, semantic, and episodic (Gold, Balota, Kirchhoff, \& Buckner, 2005). The current data do not rule an anterior-posterior distinction out, but they do suggest that it may only apply within the domain of linguistic representations evoked by the probes. That is, the idea that midventrolateral $\mathrm{pFC}$ responds quite generally to interference among linguistic representations, whereas the anterior ventrolateral $\mathrm{pFC}$ is involved in controlled semantic retrieval, would be compatible with the current finding that differential responding was not observed here using nonmeaningful materials. That said, the current findings do rule out the hypothesis that the LVPFC operates directly on interfering episodic representations, and thus, the general selection account of midventrolateral pFC would need to be confined to selection among linguistic and not episodic representations (cf. Gold \& Buckner, 2002).

In conclusion, the current findings support a role for LVPFC in the resolution of episodic interference, but the role is indirect. This region, in conjunction with the MTG, appears to support a semantic elaboration strategy performed on the probes in response to interfering episodic representations (or the anticipation of episodic interference), and the current caudate findings further suggest that it might be useful to view this strategy as an instrumental behavior motivated by the expectation that it will facilitate successful retrieval outcomes (O’Doherty et al., 2004) or as a strategy that requires working memory updating of the different semantic representations of the probes, in light of recovered episodic evidence, during the course of the trial (Marklund et al., 2009).

\section{Acknowledgments}

This work was supported by the National Institutes of Health (MH073982 to I. G. D.) and by the Basic Science Research Program through the National Research Foundation of Korea (NRF) by the Ministry of Education, Science, \& Technology (2011-0005751 to S. H.).

Reprint requests should be sent to Sanghoon Han, Department of Psychology, Yonsei University, Seoul, South Korea, or via e-mail: sanghoon.han@yonsei.ac.kr.

\section{REFERENCES}

Alexander, G. E., DeLong, M. R., \& Strick, P. L. (1986). Parallel organization of functionally segregated circuits linking basal ganglia and cortex. Annual Review of Neuroscience, 9, 357-381.

Badre, D., Poldrack, R. A., Pare-Blagoev, E. J., Insler, R. Z., \& Wagner, A. D. (2005). Dissociable controlled retrieval and generalized selection mechanisms in ventrolateral prefrontal cortex. Neuron, 47, 907-918.

Badre, D., \& Wagner, A. D. (2007). Left ventrolateral prefrontal cortex and the cognitive control of memory. Neuropsychologia, 45, 2883-2901.

Barnes, K. A., Cohen, A. L., Power, J. D., Nelson, S. M., Dosenbach, Y. B., Miezin, F. M., et al. (2010). Identifying basal ganglia divisions in individuals using resting-state functional connectivity MRI. Frontiers in Systems Neuroscience, 4, 18.

Binder, J. R., Desai, R. H., Graves, W. W., \& Conant, L. L. (2009). Where is the semantic system? A critical review and meta-analysis of 120 functional neuroimaging studies. Cerebral Cortex, 19, 2767-2796. 
Borst, J. P., Taatgen, N. A., Stocco, A., \& van Rijn, H. (2010). The neural correlates of problem states: Testing fMRI predictions of a computational model of multitasking. PLOS ONE, 5, e12966.

Brett, M., Anton, J.-L., Valabregue, R., \& Poline, J.-B. (2002) Region of interest analysis using an SPM toolbox. Paper presented at the 8th International Conference on Functional Mapping of Human Brain, Sendai, Japan.

Cadoret, G., \& Petrides, M. (2007). Ventrolateral prefrontal neuronal activity related to active controlled memory retrieval in nonhuman primates. Cerebral Cortex, 17(Suppl. 1), i27-i40.

Dobbins, I. G., Foley, H., Schacter, D. L., \& Wagner, A. D. (2002). Executive control during episodic retrieval: Multiple prefrontal processes subserve source memory. Neuron, 35, 989-996.

Dobbins, I. G., \& Han, S. (2006). Cue- versus probe-dependent prefrontal cortex activity during contextual remembering. Iournal of Cognitive Neuroscience, 18, 1439-1452.

Dobbins, I. G., \& Wagner, A. D. (2005). Domain-general and domain-sensitive prefrontal mechanisms for recollecting events and detecting novelty. Cerebral Cortex, 15, $1768-1778$.

Fox, M. D., Snyder, A. Z., Vincent, J. L., Corbetta, M., Van Essen, D. C., \& Raichle, M. E. (2005). The human brain is intrinsically organized into dynamic, anticorrelated functional networks. Proceedings of the National Academv of Sciences. U.S.A., 102, 9673-9678.

Gerbella, M., Belmalih, A., Borra, E., Rozzi, S., \& Luppino, G. (2010). Cortical connections of the macaque caudal ventrolateral prefrontal areas $45 \mathrm{~A}$ and 45B. Cerebral Cortex, 20, 141-168.

Gold, B. T., Balota, D. A., Kirchhoff, B. A., \& Buckner, R. L. (2005). Common and dissociable activation patterns associated with controlled semantic and phonological processing: Evidence from fMRI adaptation. Cerebral Cortex, 15, 1438-1450.

Gold, B. T., \& Buckner, R. L. (2002). Common prefrontal regions coactivate with dissociable posterior regions during controlled semantic and phonological tasks. Neuron, 35, 803-812.

Grahn, J. A., Parkinson, J., \& Owen, A. M. (2008). The cognitive functions of the caudate nucleus. Progress in Neurobiology, 86, 141-155.

Jacoby, L. L., Shimizu, Y., Daniels, K. A., \& Rhodes, M. G. (2005). Modes of cognitive control in recognition and source memory: Depth of retrieval. Psvchonomic Bulletin E Review, 12, 852-857.

Law, J. R., Flanery, M. A., Wirth, S., Yanike, M., Smith, A. C., Frank, L. M., et al. (2005). Functional magnetic resonance imaging activity during the gradual acquisition and expression of paired-associate memory. The lournal of Neuroscience, 25, 5720-5729.

Marklund, P., Larsson, A., Elgh, E., Linder, J., Riklund, K. A., Forsgren, L., et al. (2009). Temporal dynamics of basal ganglia under-recruitment in Parkinson's disease: Transient caudate abnormalities during updating of working memory. Brain, 132, 336-346.

Miyashita, Y., Higuchi, S. I., Sakai, K., \& Masui, N. (1991). Generation of fractal patterns for probing the visual memory. Neuroscience Research, 12, 307-311.
Norman, D. A., \& Bobrow, D. G. (1979). Descriptions: An intermediate stage in memory retrieval. Cognitive Psvchologv, 11, 107-123.

O’Doherty, J., Dayan, P., Schultz, J., Deichmann, R., Friston, K., \& Dolan, R. J. (2004). Dissociable roles of ventral and dorsal striatum in instrumental conditioning. Science. 304, 452-454

Paivio, A. (1991). Dual coding theory: Retrospect and current status. Current Journal of Psychology, 45, 255-287.

Petersen, S. E., Fox, P. T., Posner, M. I., Mintum, M., \& Raichle, M. E. (1988). Positron emission tomographic studies of the cortical anatomy of single-word processing. Nature, 331, $585-589$

Raposo, A., Han, S., \& Dobbins, I. G. (2009). Ventrolateral prefrontal cortex and self-initiated semantic elaboration during memory retrieval. Neuropsychologia, 47, 2261-2271.

Roediger, H. L. (1990). Implicit memory: Retention without remembering. American Psvchologist, 45, 1043-1056.

Thompson-Schill, S., D’Esposito, M., Aguirre, G., \& Farah, M. (1997). Role of left inferior prefrontal cortex in retrieval of semantic knowledge: A reevaluation. Proceedings of the National Academv of Sciences. U.S.A., 94, 14792-14797.

Thompson-Schill, S. L. (2003). Neuroimaging studies of semantic memory: Inferring "how" from "where". Neuropsychologia, 41, 280-292.

Tulving, E. (1983). Elements of episodic memory. Oxford, U.K.: Oxford University Press.

Tulving, E., \& Thomson, D. M. (1973). Encoding specificity and retrieval processes in episodic memory. Psvchological Review, 80, 359-380.

Van Dijk, K. R. A., Hedden, T., Venkataraman, A., Evans, K. C., Lazar, S. W., \& Buckner, R. L. (2010). Intrinsic functional connectivity as a tool for human connectomics: Theory, properties, and optimization. Journal of Neurophysiology, 103, 297-321.

Vandenberghe, R., Price, C., Wise, R., Josephs, O., \& Frackowiak, R. S. J. (1996). Functional anatomy of a common semantic system for words and pictures. Nature, 383, 254-256.

Veltman, D. J., Rombouts, S. A., \& Dolan, R. J. (2003). Maintenance versus manipulation in verbal working memory revisited: An fMRI study. Neuroimage, 18, 247-256

Wager, T. D., \& Nichols, T. E. (2003). Optimization of experimental design in fMRI: A general framework using a genetic algorithm. Neuroimage, 18, 293-309.

Wagner, A. D., Paré-Blagoev, E. J., Clark, J., \& Poldrack, R. A. (2001). Recovering meaning: Left prefrontal cortex guides controlled semantic retrieval. Neuron, 31, 329-338.

Webster, M. J., Bachevalier, J., \& Ungerleider, L. G. (1993). Subcortical connections of inferior temporal areas TE and TEO in macaque monkeys. Journal of Comparative Neurology, 335, 73-91.

Webster, M. J., Bachevalier, J., \& Ungerleider, L. G. (1994). Connections of inferior temporal areas TEO and TE with parietal and frontal cortex in macaque monkeys. Cerebral Cortex, 4, 470-483. 